\title{
A Case Study of Achalasia of Cardia
}

\author{
Md. Rafiqul Islam *1, Kazi Moinur Rahman ${ }^{2}$, Md. Showkat Ali ${ }^{3}$, S M Golam Azam ${ }^{4}$
}

\begin{abstract}
Introduction: Achalasia is a benign idiopathic disorder caused by progressive neuronal degeneration in the mesenteric plexus of Auerbach, which causing non-relaxing, hypertensive lower esophageal sphincter (LES) and aperistalsis of the esophageal body. This functional damage is irreversible and the treatment of esophageal achalasia is mainly palliative. Case Report: Our patient is a 55 years old lady presents with progressive dysphagia, regurgitation, chest pain and weight loss. After clinical examination and relevant investigations, she was diagnosed as a case of achalasia of cardia of oesophagus. Discussion: She was prepared for Heller's myotomy with antireflux procedure. Operative procedure was done under general anaesthesia. Conclusion: Postoperative recovery was good. The patient is asymptomatic, general condition improved and found healthy on follow up after one year.

Keywords: Achalasia, Laparoscopic myotomy.

Number of Figures: 02; Number of References: 05; Number of Correspondences: 04
\end{abstract}

*1. Corresponding Author: Dr. Md. Rafiqul Islam FCPS (Surgery)

Senior Consultant

Department of Surgery

General Hospital Khulna

Email: dr.rafiqrmc23@gmail.com

2. Dr. Kazi Moinur Rahman

MS (Ortho Surgery)

Senior Consultant

Department of Ortho-Surgery

General Hospital Khulna

3. Dr. Md. Showkat Ali

FCPS (Surgery)

Junior Consultant

Department of Surgery

Upozilla Health Complex, Digholia, Khulna.

4. Dr. S M Golam Azam

MS (Surgery)

Junior Consultant

Department of Surgery

Upozilla Health Complex, Rupsha, Khulna.

\section{Introduction}

Achalasia is a benign idiopathic disorder caused by progressive neuronal degeneration in the mesenteric plexus of Auerbach, which causing non-relaxing, hypertensive lower esophageal sphincter (LES) and aperistalsis of the esophageal body ${ }^{1}$. This functional damage is irreversible and the treatment of esophageal achalasia is mainly palliative. The most recognized clinical features of the disease are progressive dysphagia, regurgitation, chest pain, and weight loss ${ }^{2}$. Repeated aspiration pneumonia can be one of the clinical presentations. Achalasia is also a risk factor for the development of esophageal carcinoma, with 140-fold increased risk of cancer compared to general population ${ }^{1}$.

\section{Case Report:}

A 55 years old lady, presented with progressive dysphagia for 6 months, she complained of difficulty in swallowing food since 6 months ago, started with solid food. Besides, she also had loss of appetite and loss of weight (10 kg over the last 2 months).There was no significant past surgical history. She is a housewife and non-smoker. Clinically she was pale, dehydrated, heart rate $80 / \mathrm{min}$ (regular) and blood pressure $120 / 80 \mathrm{mmHg}$. Her weight was $45 \mathrm{~kg}$. There was no leg edema. Abdominal examination was unremarkable except for mild tenderness over epigastric region. Lungs were clear with no crepitation bilaterally. Other systemic examinations were unremarkable.

Her haemoglobin level was $12.9 \mathrm{~g} / \mathrm{dl}$; white cell count was 7,700/dl. Serum sodium was $138 \mathrm{mmol} / \mathrm{L}$, potassium level was $3.5 \mathrm{mmol} / \mathrm{L}$. Oesophago-gastric-duodenoscopy (OGDS) revealed residual food particles accumulated at the lower esophagus, with dilated lower esophagus and generalized whitish lesions (Fig. 1).

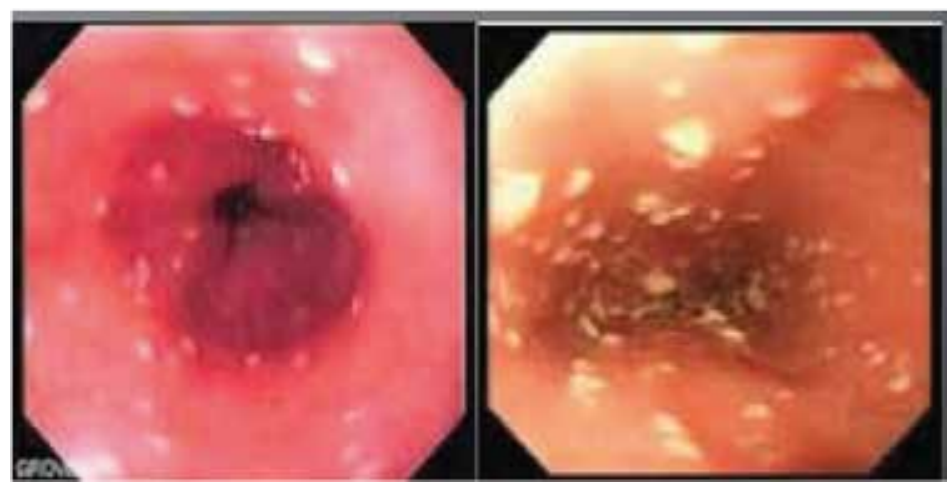

Figure-1: Oesophagial candidiasis.

There was also severe gastritis with bile reflux and mild duodenitis.

Biopsy of the lower esophagus reported as there is scattered yeast like fungal bodies with budding are seen with positive PAS and GMS staining. 
She was initially started with proton pump inhibitor (Pantoprazole). Nystatin suspension was added to treat the fungal esophagitis. Barium swallow x- ray of oesophagus (Fig. 2) showing bird's beak appearance, which strongly suggest that the patient is suffering from achalasia of cardia.

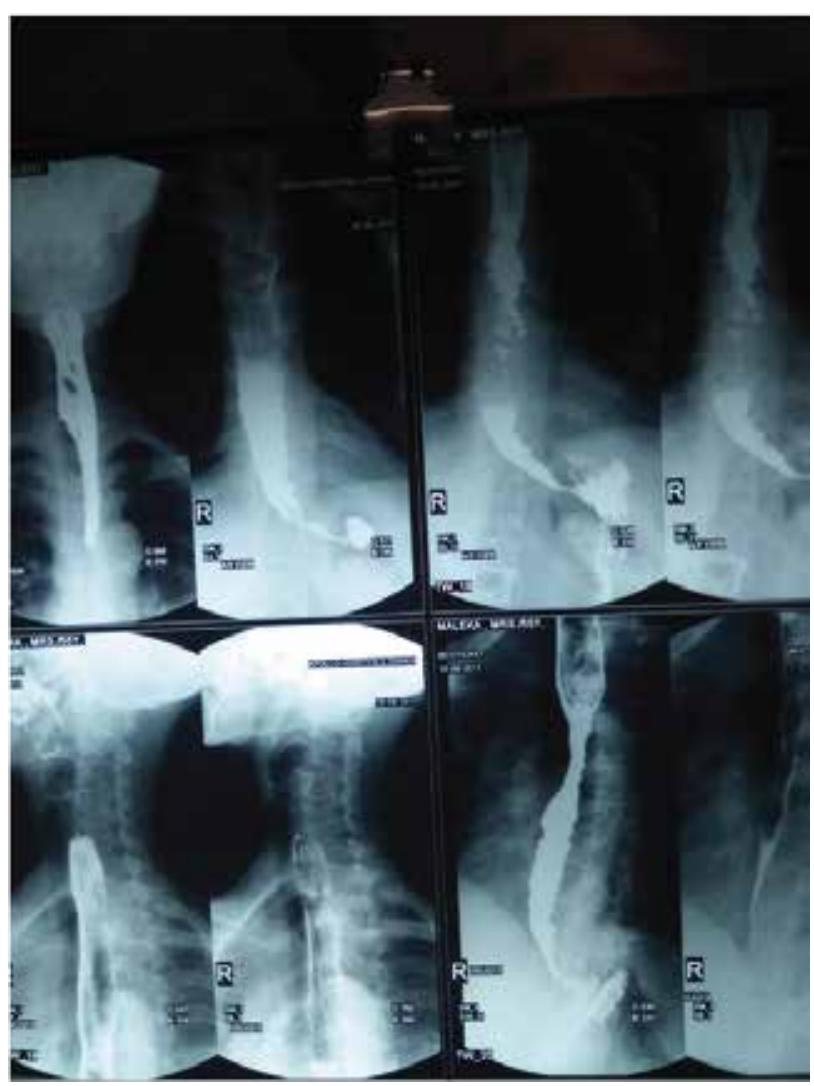

Figure-2: Barium swallow x-ray of oesophagus showing Bird' beak appearance.

After initial management, patient was taken for operative procedure. Laparotomy was done under general anaesthesia, stomach and oesophagus were mobilized, oesophagialmyotomy with anterior fundoplication (Heller-Dor's operation) was done.

Post-operative recovery was good. The general condition of the patient was gradually improving. Follow up after one year; she was healthy, having no complication and regain about five kilogram of body weight.

\section{Discussion}

There are currently four modalities for the treatment of achalasia, which including pharmacological therapy, endoscopic Botulinum toxin A (Botox) injection, pneumatic dilatation and surgical myotomy ${ }^{3}$.

Pharmacological therapy, which include isosorbitedinitrate and calcium channel blockers are generally is consideredonly in patients who are not candidate for surgery or other treatment modalities.

Endoscopic pneumatic dilatation of the LES may be the most effective treatment for achalasia non-surgically. However, it can lead to LES incompetence and gastroesophageal reflux in some cases, and the most severe complication is esophageal perforation ${ }^{4}$.

The advantage of surgical myotomy is that disruption of the muscular fibers is accomplished under direct vision. An additional procedure -anti-reflux procedure can be added during the same setting. We have done open laparotomy, then mobilization of lower oesophagus and stomach followed by a long incision at lower oesophagus (myotomy). Anterior fundoplication was done as an anti-reflux procedure.

The introduction of a laparoscopic approach (minimally invasive) has regained primacy ${ }^{5}$.

A recent meta-analysis suggested that good clinical efficacy combined with low morbidity has established laparoscopic myotomy as a definitive, safe, and effective alternative in the treatment of achalasia.

Laparoscopic myotomy is less invasive than open surgery,but is expensive and the results depend mainly on the surgeon's expertise.

Nevertheless, we strongly suggest that endoscopic treatment is advisable with frequent follow-up before surgical treatment commenced ${ }^{3}$.

\section{Conclusion}

Open surgical procedure gives good result even in long term follow up. But laparoscopic myotomy is the preferred and recommended method in the management of achalasia. Laparoscopic myotomy with partial fundoplication is superior to other treatment methods because of its excellent efficacy and safety, and also prevent acid exposure in the distal esophagus. Open surgical procedure can be performed where laparoscopic facility as well as expert personal is not available.

\section{Consent:}

Prior informed consent was obtained from the patient for publication of this case report and any accompanying images.

Conflict of Interests: None.

\section{Acknowledgement}

The authors gratefully acknowledge Dr.Md.Ridwanul Islam MBBS for helping with valuable suggestions and documentations.

\section{References}

1. Cameron, J.L. \& Cameron, A.M. Current surgical Therapy: Expert Consult. 10th ed. Elselvier Saunder; 2011.

2. Vaezi, M. F., Richter, J. E. Current Therapies for Achalasis: Comparison and Efficacy. Journal of Clinical Gastroenterology. 1998; 27(1): 21-35.

https://doi.org/10.1097/00004836-199807000-00006

PMid:9706766

3. Andee D, Zakaria L S, Bob S H, Nizam H, Maya M. Treatment Modalities for Achalasia Cardia: A Case Study and Literature Review. Intentional journal of case Reports in Medicine. 2013; Article ID 602167. 
4. Kostic, S., Johnsson, E., Kjellin, A., et al. Health Economic Evaluation of Therapeutic Strategies in Patients with Idiopathic Achalasia: Result of Randomized Trial, Comparing Pneumatic Dilatation with Laparoscopic Cardiomyotomy. Surgical Endoscopy. 2007a; 21(7):1184-9.

https://doi.org/10.1007/s00464-007-9310-0

PMid:17514399
5. Rosemurgy, A., Villadolid, D., Thometz. D., Kalipersad, et al. Laparoscopic Heller Myotomy Provides Durable Relief from Achalasia and Salvages Failures after botox or Dilatation. Annals of Surgery. 2005; 241(5): 725-33; discussion 733-5.

https://doi.org/10.1097/01.sla.0000160702.31452.d5

PMid:15849508 PMCid:PMC1357127 